\title{
Construction of Electronic Scoring System for Competitive Martial Arts Based on RFID and Face Recognition Technology
}

\author{
Guofang Kuang ${ }^{1}$, Chunlin Kuang ${ }^{1}$ and Hongsheng $\mathrm{Xu}^{1,2} \mathrm{a}^{*}$ \\ ${ }^{1}$ Luoyang Normal University, Luoyang, 471934, China \\ ${ }^{2}$ Henan key Laboratory for Big Data Processing \& Analytics of Electronic Commerce, Luoyang, \\ 471934, China \\ a85660190@qq.com
}

Keywords: RFID; Face recognition; Electronic scoring; Competitive martial arts; Judge

\begin{abstract}
When people want to give a mark for athlete of competitive martial arts, Ten Judges are needed. These Judges are divided into three groups. Each group give his score from different point of view According to the rules. In this paper, these characteristics of rules about competitive martial arts are analyzed. By introducing face recognition and RFID into the system firstly and combining with WLAN technology, a set of economic, practical, safe and reliable scoring system is designed. The paper presents construction of electronic scoring system for competitive martial arts based on RFID and face recognition technology.
\end{abstract}

\section{Introduction}

In 1958, China promulgated the first set of martial arts competition rules, which marked the birth of competitive martial arts and solved the problem of martial arts routine. Competitive martial arts have gradually formed cultural characteristics different from traditional martial arts. It is more standardized, unified, and focused on innovation. Competitive martial arts also play an important role in the modern transformation of martial arts. In 2012, the new martial arts competition rules were issued. From 1959 to 2012, the martial arts competition rules were revised more than ten times successively. Its scoring system and its contents were constantly evolving, gradually from the division estimation to the quantification [1]. Competitive martial arts routine evaluation becomes materialize, objective and scientific.

Since the 2012 rule of martial arts routine Competition were promulgated, The evaluation system based on the action quality, exercise levels, difficulty level and difficulty of action quality has already formed. On the one hand, this rule greatly improves the objectivity of competitive martial arts; on the other hand, it also increases the complexity for Judge. For example, starting from the 2003 edition of the competition rules, the evaluation index is coded one by one. This lays the foundation for the computerization of the scoring system.

At the end of each match, it is also possible to study the score which stored in database by judges. Judge can know their Scoring ability by analyzing the accuracy of score. By this method, judges' law enforcement level can improved, and the role of education, police and supervisory to judge also can be reduced. This system can provide the data for the management of martial arts Management Center in selecting judges.

In this paper, a convenient and reliable modern electronic scoring system is constructed by using tablet computer, combining with WLAN and identity recognition technology. The system can realize fast pre-match recording, real-time scoring, post competition information releasing and competition data analyzing. The realization of the system can further improve the modern level of martial arts match, make the martial arts match more fair and just, and promote the development of Chinese martial arts. 


\section{Analysis of Key Techniques in RFID-WLAN and Face Recognition}

In the field of computer vision, the rapid development of face recognition technology can not be separated from the improvement of computer performance, which provides computing conditions for complex graphics computing [2]. Face recognition is based on the face information to obtain facial features by the image processing, and then through a specific algorithm to match and recognize facial features. From analyzing the face recognition process, the whole system is made of video input module, image preprocessing module, face detection module, feature extraction, recognition matching module and output module. Each module in face recognition is shown in figure 1.

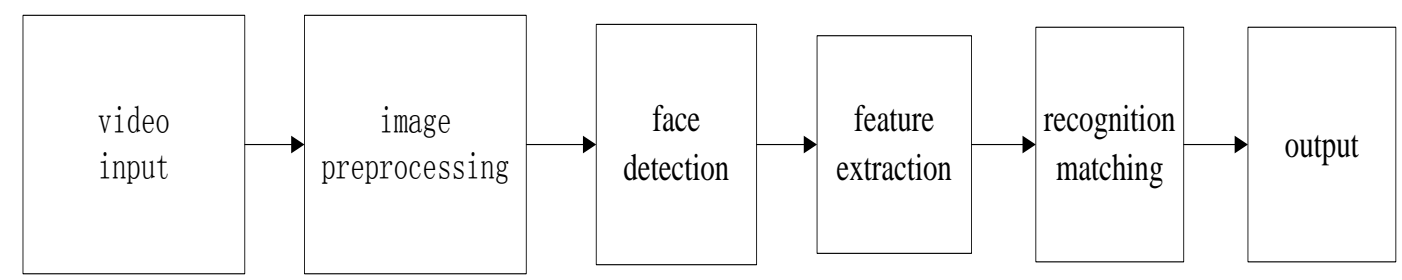

Figure 1. Modules of Recognition

Video input is a prerequisite for recognition. The other factors, such as background change, makeup, facial expression change, will affect the detection of human faces.In practical applications, the factors that affect video are also different [3]. For example, too much light in indoor lighting leads to insufficient light in foreground images, and detecting foreground targets is Difficulty because background objects are complex and similar to foreground targets. It is necessary to select the best image processing algorithm combined with the environment of the actual system.

Feature extraction refers to the feature which can distinguish the face from the non-face. The face feature extraction is often affected by the algorithm model and image quality.

The basic calculation of LBP operator is expanded on a $3 \times 3$ rectangle. Take the center point of the rectangle as the base point, and find all the pixel values in the nearby area to compare to them. The smaller position than the base point is set to 0 . Instead, the value larger than the base point is set to 1 , and the comparison results are sorted [4]. Then the quantization results are weighted and added according to different positions, and the final value is the value corresponding to the local binary mode of the center point. Assume that the central pixel value is $P_{C}$, the pixel value of the other eight positions adjacent to it is $\mathrm{P} 1, \mathrm{P} 2, \cdots \cdots \mathrm{P} 8$, as shown in formula 1 for any LBP operator:

$$
\mathrm{LBP}_{\mathrm{P}_{\mathrm{C}}}=\sum_{i-1}^{S} S\left(P_{c}-P_{i}\right) \times 2^{i-1}, S(x)=\left\{\begin{array}{l}
1, x \leq 0 \\
0, x>0
\end{array}\right.
$$

The image is scanned line by line according to the LBP operator.The LBP coding of each pixel is obtained, and the LBP response image is generated [5]. Then the LBP image is divided into several regions, and the LBP coding histogram of each region is obtained. Then the LBP coded histogram of the whole image is obtained, which is called the LBP feature. The LBP feature of the response image include information about the distribution of pixels, edges, and local features. Because the LBP operator is an algorithm derived from the definition of texture local proximity, the calculation of the LBP value of each pixel value depends on the surrounding pixel value. It is more favorable for the extraction of texture features to preserve the details.

WLAN utilizes electromagnetic wave, wireless medium, to transmit data, so it not only reduces requirements and expenses for network wiring, but also provides users an information-obtaining approach with more flexibility and mobility. In this system, the data transmitted includes both non-media data that requires a certain rate and media data that requires a higher rate.

Currently, Five main criteria are typically used in WLAN:IEEE 802.11b, IEEE 802.11g,IEEE $802.11 \mathrm{n}$ and IEEE $802.11 \mathrm{ac}$ [6]. The transmission rate of IEEE $802.11 \mathrm{~b} / \mathrm{g} / \mathrm{n}$ is vary from $11 \mathrm{Mbps}$ to $475 \mathrm{Mbps}$. Working in the $5 \mathrm{G}$ band, the transmission rate of IEEE 802.11 ac can theoretically provide 
data transmission capacity up to $1 \mathrm{Gbit}$ per second. Therefore, there is no problem in choosing WLAN to build an electronic scoring system in terms of data transmission speed.

Network reliability is more important for this system because all data and commands transmitted should be right to satisfy the demand of judging work. Otherwise, it is valueless for this system. Some techniques implemented in WLAN, such as spread spectrum, ensure the security and reliability of data transmitting. Bluetooth is a technology about WLAN. But essentially it is a wire-replaced technology with low power, small area and low rate. Generally, its transmitting rate is about between $10 \mathrm{Kbps}$ and $1 \mathrm{Mbps}$, and transmitting distance is from 10metres to 100metres. It is not appropriate for application of the system on transmitting rate and distance. For these drawbacks, Bluetooth is excluded.

Radio Frequency Identification (RFID) is a non-contact automatic identification technology, which is based on the transmission characteristics of radio frequency signals or spatial coupling (inductance or electromagnetic coupling). Compared with other automatic identification techniques (bar code, optical identification and biometrics, including iris, face, sound and fingerprint), RFID has strong anti-interference ability and large amount of information. Because of the characters about non-visual range reading, writing and long life, RFID are widely used in logistics, supply chains, animal and vehicle identifications, access control systems, book managements, automatic charges and manufacturing fields [7].

According to the basic working mode of RFID system, it can be divided into full duplex system , half duplex system and timing system. In full duplex and half duplex systems, because the response signals of tags are very weak compared with the signals sent by readers, it is necessary to use appropriate transmission methods to distinguish the reply signals of tags and the query signals of readers.

\section{Construction of Electronic Scoring System for Competitive Martial arts}

System hardware consists of ten parts. Their are data center, technician equipment, judge equipment, information publishing equipment,superior judge equipment, supervisory equipment, arbitration equipment, checking equipment, and video equipment and wireless communication equipment . These parts are shown in figure 2 .

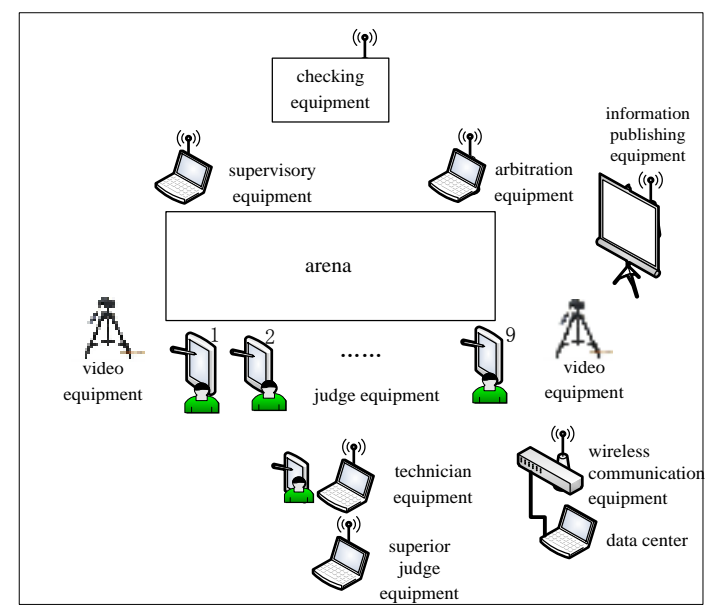

Figure 2. System hardware structure

(1) Data center plays an important role in the system. It acts as database server. All athlete information and competition data are stored in it.

(2) Technician equipment is the core component in the system. All devices have to communicate with it. When Technician receives the beginning command of competition, he will press the button on the equipment. The competition is controlled by it, until competition ending. Technician equipment reads data from the data center and sends it to other equipment other than the checking device.

(3) Judging terminals mainly receive the data from technician equipment, and mark them in terms of judging criteria and rules. Then, the scores are sent and saved into the database. The quantity of judging terminals varies in different items. 
(4) Information publishing equipment is used to release the results dynamically, and it provides the newest detail of competition for all the people in the match.

(5) Superior judge equipment serves for superior judge. By it, superior judge can keep track of judges' scoring, modify the total score within the limits of his own authority, and control the progress of the match.

(6) Supervisory equipment is used by supervisory personnel. Track of judges' scoring can be know from these equipment.

(7) Arbitration equipment is Arbitration personnel. If a complaint is made, the arbiter makes an award based on the data of the equipment.

(8) Checking equipment includes RFID reader, image capture equipment and image recognition equipment.Using RFID reader to read the identity information of the athletes, and using the image capture device to obtain the face information of the participants, the recognition device matches the identity information read by RFID with the obtained face information, and finally outputs the result of checking. The component of these equipment is as shown in figure 3.

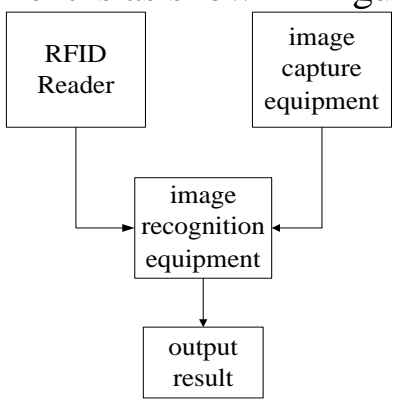

Figure 3. Checking equipment

(9) Video equipment is used to record video for current athlete. And the video file is saved in the computer. Every element performed by current athlete is recorded witch certain name. It is prepared for manager of the match to position the video easily and replay the chosen elements' clips. In order to replay smoothly.

(10)Wireless communication equipment use wireless signals to communicate with other equipment.It is the intermediate node for information transmission.

\section{Main Data Structure and Communication Protocol}

The communication center of data is technician, and the technician module initiates the communication connection to other modules. In response to the communication connection, the other modules establish the communication connection and begin the data exchange. The communication connection between technician and each module is stored in a key-value pair data structure. The primary key is the code name of each module, and the client information is stored in the key value. In the communications, the primary key will be used to find the client. The structure of the client and key-value pair structure is shown in figure 4.

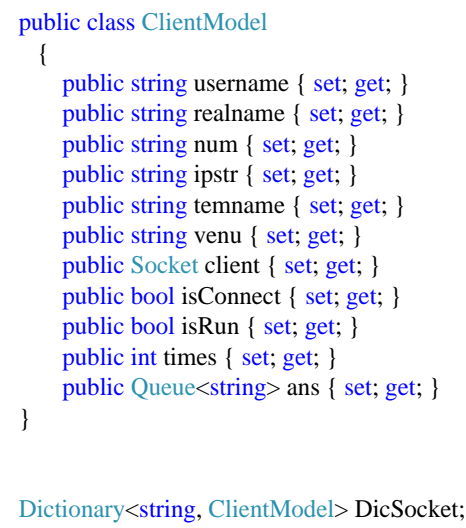

Figure 4. The main data structure 
Client Model is the model, in which the fields represent users' name, real name, judge's number, IP address, terminal name, venue, communication connection, whether to connect, whether to run, connection time and data sending queue. DicSocket is the model of the key value pair.

The data exchange of the system is based on Json format. The data packet exchange is centered on the technician module. The data received by the data center can be divided into data information and control instructions. Data information mainly includes user login information, referee scoring information and athlete status. User login information includes user name and password; referee scoring information includes scoring information of group A, group B and group C; athletes' status includes normal, irregular and incomplete. Control order refers to the control order generated by the chief referee module, including timing start, timing end and whether to allow the referee to confirm. The data sent by the data center is mainly sent by the referee, the chief referee sends out the control order and the control order issued by himself.

\section{Summary}

This paper summarizes the modern electronic scoring system of competitive Wushu, introduces the key technology of the system, and describes the architecture and software implementation of the system. The system is not only convenient to score, but also can analyze the competition data. Guide martial arts training. The system can also be extended to the traditional Wushu competition electronic scoring system, the use of a system to easily achieve two functions, these extensions will be implemented in the future software upgrade.

\section{Acknowledgements}

This paper is supported by Science and Technology Key Project of Henan Province (162102310478), and by School Applied Science and Technology Research Foundation 2017 "Research on the Application of Wushu (routine) Intelligent Competition arrangement system based on SVM-KNN".

\section{References}

[1] Shao Kui-hua, Research on the Model of athlete selection and Evaluation based on sudden change Series, Journal of Shandong Institute of physical Education. 2012,28(1).

[2] Yan Bo, Development of traceability platform for aquatic product supply chain based on RFID and EPC, Journal of Agricultural Engineering. 2013,29(5).

[3] Joe Kman, Wang Guiran, Sun Wei, et al, Perceived Sports: the Application Architecture of Internet of things Technology in Sports, Journal of Wuhan Institute of physical Education. 2012, 46(1).

[4] Zhang Hailiang, The birth of competitive Wushu, Shanghai: Shanghai Institute of physical Education.2010.

[5] Kuang Guofang, The Application of Management Information System in Internet of Things based on RFID Technology,Sensors\&Transducers. 2013.7.

[6] Xiangru Xu,Research And Implementation Of The Authentication System Based on Face Recognition,Chengdu:University of Electronic Science and Technology of China.2016.

[7] Yang Gao,A research on Face Detection and Recognition Algorithms, Taiyuan:North University Of China. 2015. 